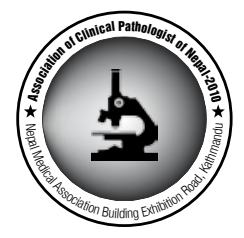

\title{
Kikuchi-Fujimoto disease
}

\section{Adhikari $\mathrm{RC}^{1}$}

${ }^{1}$ Department of Pathology, Tribhuvan University Teaching Hospital, Kathmandu, Nepal

\author{
Keywords: \\ Cytopathology; \\ Histopathology; \\ Kukuchi-Fujimoto disease
}

\begin{abstract}
Kikuchi-Fujimoto disease or histiocytic necrotizing lymphadenitis is a benign, self limited condition with higher prevalence among Japanese and other Asiatic people. Though the cause of this disease remains unclear, viral cause has been suggested. It is clinically characterized by lymphadenopathy, fever, cutaneous erythema, diarrhea, vomiting, sore throat, arthralgia, myalgia and hepatosplenomegaly. Laboratory findings are non-specific and Kikuchi-Fujimoto disease is generally diagnosed based on characteristic histopathological findings. Affected lymph nodes demonstrate paracortical areas of apoptotic necrosis with abundant karyorrhectic debris, proliferation of histiocytes, plasmacytoid monocytes, small and transformed lymphocytes in the absence of neutrophils. Kikuchi-Fujimoto disease is thought to have three evolving phases: proliferative, necrotizing and xanthomatous. Fine needle aspiration smears from involved lymph nodes reveal characteristic intra- and extracellular apoptotic nuclear debris with admixed crescentic macrophages on a reactive lymphoid background. Differential diagnoses of this disease are lymphoma, systemic lupus erythematosus, toxoplasmosis, tuberculosis, myeloid tumor and even metastatic adenocarcinoma. Treatment is symptomatic and spontaneous recovery occurs in 1 to 4 months.
\end{abstract}

\section{INTRODUCTION}

Kikuchi-Fujimoto disease (KFD), an enigmatic, benign disorder, was first described in 1972 simultaneously by Kikuchi $^{1}$ and Fujimoto and colleagues ${ }^{2}$ and is now recognized worldwide. It is a rare cause of lymphadenopathy, predominantly affecting young women with a predilection for cervical lymphadenopathy. Despite many reports and studies, the etiology of this disease remains unclear, however viral or autoimmune cause has been suggested. The disease manifests with cervical lymphadenopathy, fever or flu-like symptoms, malaise, weight loss, loss of appetite, nausea, vomiting, diarrhea, chest pain, splenomegaly and hepatomegaly. ${ }^{3,4}$ Laboratory tests are non-specific and they are anemia, elevated erythrocyte sedimentation rate (ESR), neutropenia, relative lymphocytosis and atypical

\author{
Correspondence: \\ Dr. Ram Chandra Adhikari, $M D$ \\ Consultant Pathologist, Department of Pathology, Om Hospital \& Research \\ Centre, Kathmandu, Nepal \\ GPO Box: 2496 \\ E-mail: rcadhikari@hotmail.com
}

lymphocytosis. ${ }^{5}$

The diagnosis of KFD is established by identifying characteristic histopathologic features in the lymph node; however, morphological features may simulate systemic lupus erythematosus (SLE), Non-Hodgkin lymphoma (NHL), and reactive lymphadenopathy of other causes. The intermingling of distinctive crescentic histiocytes, karyorrhectic debris, and plasmacytoid monocytes in the form of nodules and the paucity of neutrophils are consistent findings that permit a diagnosis of KFD. ${ }^{6}$ This study briefly reviews KFD with emphasis on the histopathological, cytopathological findings and differential diagnoses.

\section{DISCUSSION}

\section{Epidemiology}

KFD is known to have a worldwide distribution with a higher prevalence among Japanese and other Asian people. ${ }^{7}$ Isolated cases were reported from Europe. Six cases of KFD 
were reported from Nepal in 2003. ${ }^{8}$ Affected patients most often are adults under the age of 30 years; the mean age in a study of $\mathrm{Nepal}^{8}$ and Taiwan ${ }^{9}$ was 21.67 year and 21 years respectively. A recent series from China of 138 children with KFD revealed a mean age of 9.5 years. ${ }^{10}$ The reported female-male ratio varies from $1: 1$ to $4: 1,3,5$

\section{Etiology and Pathogenesis}

Though the definite cause of KFD is not clear, a viral or autoimmune cause has been suggested. Some initial reports described toxoplasma ${ }^{11}$ and Yersinia enterocolitica ${ }^{12}$ as possible causative agents; however, subsequent studies failed to support this fact. Recently, a case was reported hypothesizing Giardia lamblia intestinalis as a new pathogen with possible link to KFD. ${ }^{13}$

Viruses described as a causative agent in KFD are EpsteinBar virus (EBV), Human herpes virus (HHV)-6, HHV-8, human T-lymphocytic virus (HTLV)-1 and parvovirus B19. The role of EBV as well as other viruses (HHV6, HHV8, parvovirus B19) in the pathogenesis of KDF remains controversial and not convincingly demonstrated. ${ }^{7}$ A viral infection is, nevertheless, possible by virtue of clinical manifestations, as described by Unger et $\mathrm{al}^{14}$ (upper respiratory prodrome, atypical lymphocytosis and lack of response to antibiotic therapy), and certain histopathologic features ( proliferation of immunoblasts, predominance of T-cells as revealed by immunologic marker studies).

Some HLA class II series are more frequent in patients with KFD. In particular, the incidence of DPA $1 * 01$ and DPB* 0202 alleles is significantly higher in patients with KFD than in healthy control subjects. ${ }^{7}$ An exuberant T cells-mediated immune response in genetically susceptible individuals to a variety of stimuli may initiate the disease.

Electron microscopic studies have identified tubular reticular structures in the cytoplasm of stimulated lymphocytes and histiocytes in patients with KDF. ${ }^{15}$ Since these structures have also been noted within endothelial cells and lymphocytes of patients with SLE and other autoimmune disorders, some authors ${ }^{16}$ hypothesized that KFD may reflect a self-limited autoimmune condition induced by virus-infected transformed lymphocytes.

The mechanism of cell death involved in KFD has not been studied extensively. The nuclear debris, present in KFD might indicate cell death by apoptosis. According to Ohshima and coworkers ${ }^{17}$, proliferating CD 8 positive T-cells may act as "killers" and "victims" in the apoptotic process via fas- and perforin pathways.

\section{Clinical manifestations}

KFD has acute or sub acute onset evolving a period of 2 to 3 weeks. The disease usually involves cervical lymph nodes and cervical lymphadenopathy is present in $56 \%$ to $98 \%$ of cases with predilection to the posterior cervical triangle. ${ }^{?}$ Involvement of axillary $^{8}$, mesenteric ${ }^{18}$, mediastinal ${ }^{3}$, retroperitoneal $^{7}$, inguinal, intraparotid, iliac, celiac, and peripancreatic ${ }^{3}$ lymph nodes have been reported as well. Generalized lymphadenopathy sometimes occurs. ${ }^{5}$ Lymph node size ranges from 0.5 to $4 \mathrm{~cm}$ and rarely larger than $6 \mathrm{~cm}$. In addition to lymphadenopathy, patients with KFD may have fever ${ }^{3,4}$, cutaneous erythema ${ }^{19}$, diarrhea, vomiting, chest pain, arthralgia, myalgia, sore throat ${ }^{14}$, night sweats ${ }^{15}$, weight loss and hepatosplanomegaly. ${ }^{3,4}$ KFD has also been reported as a cause of fever of unknown origin and systemic symptoms are found more frequently when extranodal involvement is present. ${ }^{20,21}$

\section{Laboratory findings}

Some patients with KFD have anemia and elevation of ESR. Mild leukopenia has been observed in $25 \%$ to $58 \%$ of patients whereas leukocytosis is found in $2 \%$ to $5 \%$ of cases. $^{7}$ Moreover, $25 \%$ to $31 \%$ of patients have atypical peripheral blood lymphocytes ${ }^{14,15,22}$, which might support the aforementioned speculated viral cause. In addition to anemia and leukopenia, recurrent thrombocytopenia ${ }^{10}$, increased C-reactive protein and an increased serum lactate dehydrogenase level occur. ${ }^{7}$

\section{Unusual findings and associated disease}

Involvement of extranodal sites by KFD is uncommon, but skin, eye, lung and pleura, joint, and bone marrow involvements have been reported. ${ }^{21-25}$

Skin lesions in KFD include variety of dermatological patterns like rashes, nodules, erythematous crusted papules, indurated erythematous lesions, erythema multiforme, and erythematous maculo-papular eruptions, all mainly affecting the face and upper body. ${ }^{7,19,21}$ Eye manifestation of KFD includes bilateral anterior uveitis, while lung and pleura involvement has been described as bilateral pleural effusion and interstitial lung disease.

KFD has been reported in HIV-positive patients and in association with brucellosis, systemic juvenile idiopathic arthritis, cutaneous necrotizing vasculitis and pulmonary hemorrhage. ${ }^{26-30}$ SLE has developed in some patients thought to have true KFD suggesting that KFD could be an incomplete form of an autoimmune condition. KFD can precede, postdate, or coincide with the diagnosis of SLE. ${ }^{31}$ There have been reports of unusual features of KFD including parotid gland involvement, thyroiditis, carcinoma and diffuse large B-cell lymphoma. ${ }^{32-34}$

\section{Histopathology}

KFD is diagnosed on the basis of an excisional biopsy of affected lymph nodes. Histological features are summarized 
in table $1 .{ }^{35}$ Involved lymph nodes show partially effaced architecture by paracortical nodules of apoptotic necrosis with abundant karyorrhectic debris and large numbers of histiocytes (fig. 1). The plasmacytoid monocytes tend to cluster, particularly at the margins of the necrotic foci. Admixed are many small lymphocytes and immunoblasts. Reactive immunoblastic component in some cases may be mistaken for lymphoma. Neutrophils and eosinophils are absent and plasma cells are scarce or absent. ${ }^{7}$ Thrombosed vessels may be present in regions, peripheral to necrosis. Reactive lymphoid follicles are observed in most cases. The karyorrhectic process can extend beyond the nodal capsule into perinodal tissue. ${ }^{?}$

Kuo $\mathrm{TT}^{5}$ proposed three histologic phases of KFD: proliferative, necrotizing and xanthomatous. The initial proliferative phase features an expanded paracortex with increases in various histiocytes, plasmacytoid monocytes, which are admixed with lymphocytes and nuclear debris. The necrotizing phase is characterized by presence of necrosis of any degree. If foamy histiocytes predominate in the lesions, the case is categorized as being in the xanthomatous phase despite the presence or absence of necrosis. These three histologic types of KFD could represent different evolving stages of the disease, however, this speculation has not been confirmed because of a lack of studies with sequential biopsies.

Biopsies of skin lesions from patients with KFD revealed dermal infiltration by apoptotic plasmacytoid monocytes and other cell infiltrates, similar to components of affected lymph nodes.

\section{Cytopathology}

The accurate diagnosis of KFD on fine needle aspiration cytology (FNAC) is possible on adequately sampled and well-prepared specimen with given correct clinical data. The cytological smears demonstrate characteristic intraand extracellular apoptotic nuclear debris with admixed crescentic macrophages (fig. 2) on a reactive lymphoid background.$^{36}$ In some cells, nuclei may be ragged, dense and pyknotic as the apoptosis progresses. Free nuclear fragmentation may be present in the background as small, variably sized dark staining karyorrhectic globules. Crescentic macrophages are characterized by eccentrically located nuclei compressed into a thin or buckled crescent against the cell membrane with clear cytoplasm containing apoptotic debris. Phagocytic histiocytes and monocytes have reniform nuclei. Neutrophils are notably absent and large numbers of interspersed small mature lymphocytes and some larger transformed lymphocytes are also present.

Differential diagnoses of KFD on cytological smears include SLE, NHL and reactive lymphadenitis of other causes. Lupus lymphadenitis is indistinguishable from KFD cytologically. Recognition of characteristic apoptotic nuclear

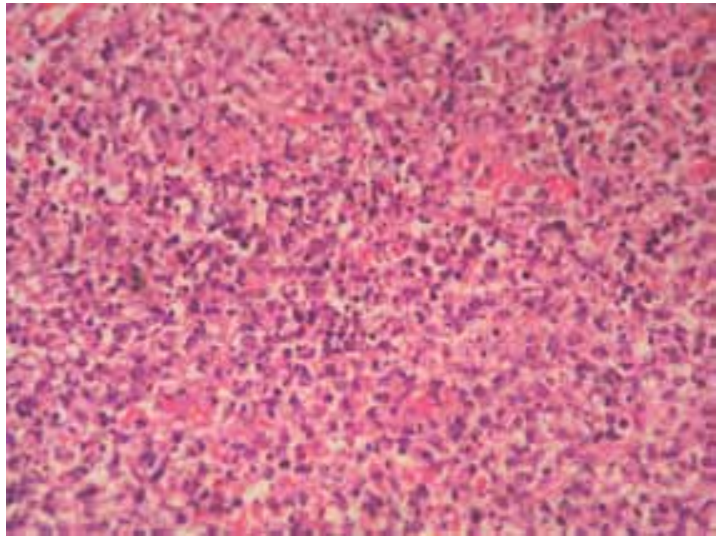

Figure 1: Lymphnode biopsy, showing apoptotic necrosis with abundant karyorrhectic debris and large numbers of histiocytes (HE stain, X200).

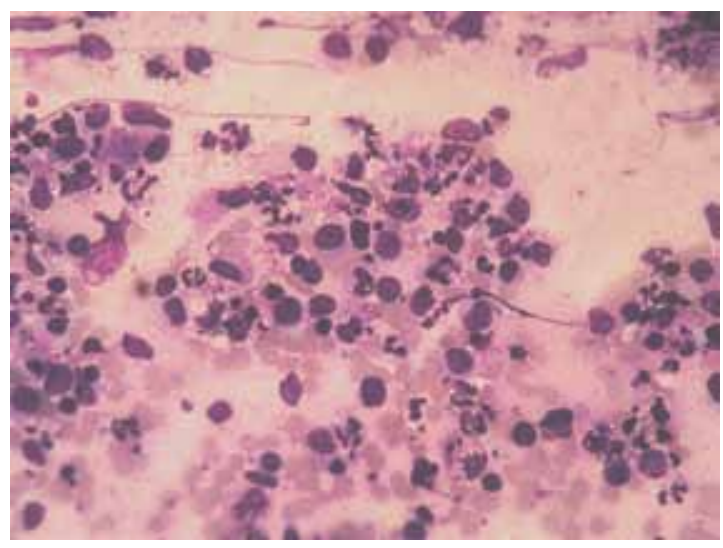

Figure 2: Lymphnode aspirate, showing intra- and extracellular apoptotic nuclear debris and macrophages (Giemsa stain, X200).

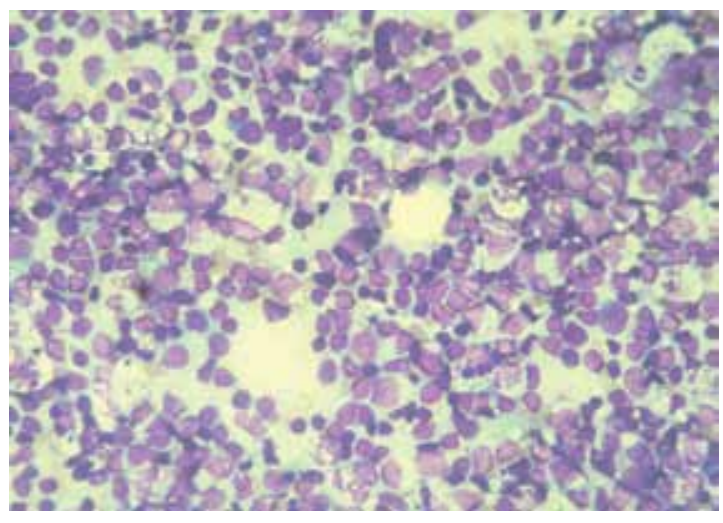

Figure 3: Lymphnode aspirate from patients with KFD, showing large number of transformed lymphoid cells, mimicking NHL (Giemsa stain, X400).

debris and crescentic macrophages helps to differentiate KFD from reactive lymphadenitis of other causes. The aspirate containing large number of transformed lymphoid cells (fig. 3) raises the suspicion of NHL. Apoptosis may occur in lymph node secondary to NHL and in these cases lymph node excision and histopathological examination may require for definite diagnosis. 


\section{Ancillary studies}

Flow cytometric analysis is helpful to exclude the possibility of NHL. KFD typically shows predominance of T cells with most being phenotypically unremarkable CD $8+$ cells in flow-cytometric analysis. ${ }^{37}$

Immunohistochemically, histiocytes express histiocytesassociated antigens such as lysozyme, myeloperoxidase (MPO) and CD68. Plasmacytoid monocytes are positive for CDC8, but not for MPO. ${ }^{7}$ In lymphocyte population, there is predominance of T-cells with very few B cells, and abundance of CD8+ T-cells over CD4+ T-cells is noted. ${ }^{7}$

\section{Differential diagnosis}

Histological differential diagnoses of KFD include lymphoma, lymphadenopathy due to autoimmune disorders primarily SLE and infectious etiologies, such as EBV, herpes simplex virus, Bartonella henselae and toxoplasmosis. ${ }^{38}$ Other differential diagnoses are plasmacytoid T-cell leukaemia, Kawasaki disease, myeloid tumor and even metastatic adenocarcinoma. ${ }^{7}$ In developing countries like Nepal, KFD should be differentiated from tuberculous lymphadenitis as well. ${ }^{8}$ Due to differences in treatment, these entities must be excluded before a diagnosis of KFD can be made.

The differentiation of KFD from SLE can sometimes be problematic because of clinical and histological similarities. Elevated levels of ANA, presence of hematoxylin bodies, Azzopardi phenomenon, sparse CD8+ T cells and abundance of plasma cells favor SLE over KFD.

Due to presence of abundant transformed lymphocytes and immunoblasts, KFD may be misdiagnosed as NHL. However, NHL lacks striking polymorphous histiocytic infiltrate, typical of KFD. Most lymphomas are B-cell lineage and among T-cell lymphomas, CD4 expression is more common than CD68, whereas a predominance of CD68 positivity is characteristic of KFD. ${ }^{7}$ Classic Hodgkin lymphoma could cause necrosis and have histiocytic infiltrate mimicking KFD. But the presence of ReedSternberg cells, which are stained with CD30 or CD15 or both, and numerous eosinophils and neutrophils make its recognition relatively easier. ${ }^{38}$

Plasmacytoid T-cell leukaemia displays T-zone expansion by plasmacytoid-like cells, later developing acute or chronic myelomonocytic leukaemia. These plasmacytoid cells do not express MPO in contrast with KFD histiocytic component that is strikingly MPO positive. $^{7}$

The distinction from tuberculous lymphadenitis is important especially in region where tuberculosis is quite common. The epithelioid cell granuloma, multinucleated giant cells of Langhans' type, caseous necrosis and absence of karyorrhectic debris clearly favor the diagnosis of tuberculosis. ${ }^{8}$ The crescentic histiocytes of KFD may resemble signet-ring cells mimicking metastatic adenocarcinoma. However, metastatic adenocarcinoma is composed of cells with atypical nuclei and contain mucin rather that cellular debris. ${ }^{7}$

\section{Disease course and management}

KFD is a self-limiting condition, usually resolving within 1 to 4 months, but a low recurrence rate of $3 \%$ to $4 \%$ has been reported. ${ }^{7}$ Rare fatal cases have been documented in the reported literature. ${ }^{39}$

There is to specific treatment for KFD due to its unknown etiology. In general, therapy is targeted toward symptomatic relief, including relief of fever and lymph node tenderness with use of analgesics and antipyretics. ${ }^{39}$ Corticosteroids are reserved for severe cases or relapsing disease. Takada $\mathrm{K}$ et al reported a case of KFD that dramatically resolved with oral minocycline treatment suggesting that the causative agent of KFD might be sensitive to this antibiotic. ${ }^{40}$

\section{CONCLUSION}

KFD is rare, self-limiting disease of unknown etiology with spontaneous remission. Although the etiology remains unclear; clinical, histological and immunohistochemical findings favor viral cause. Its diagnosis is important to prevent mismanagement as lymphoma or carcinoma and the diagnosis is made in nodal biopsy showing apoptotic necrosis with abundant karyorrhectic debris and numerous histiocytes, especially in young female with posterior cervical lymphadenopathy.

\section{REFERENCES}

1. Kikuchi M. Lymphadenitis showing focal reticulum cell hyperplasia with nuclear debris and phagocytosis. Nippon Ketsueki Gakkai Zasshi 1972;35:379-80.

2. Fujimoto Y, Kozima Y, Yamaguchi K. Cervical subacute necrotizing lymphadenitis: a new clinicopathological entity. Naika 1972;30:9207.

3. Dorfman RF, Berry GJ. Kikuchi's histiocytic necrotizing lymphadenitis: an analysis of 108 cases with emphasis on differential diagnosis. Semin Diagn Pathol 1988;5:329-45.

4. Pileri S, Kikuchi M, Helbron D, Lennert K. Histiocytic necrotizing lymphadenitis without granulocytic infiltration. Virchows Arch A Pathol Anat Histopathol 1982;395:257-71.

5. Kuo TT. Kikuchi's disease (histiocytic necrotizing lymphadenitis): a clinicopathologic study of 79 cases with an analysis of histologic subtypes, immunohistology, and DNA ploidy. Am J Surg Pathol 1995;19:798-809.

6. Server CE, Leith CP, Appenzeller J, Foucar K. Kikuchi's histiocytic necrotizing lymphadentis associated with ruptured silicone breast implant. Arch Pathol Lab Med 1996;120:380-5.

7. Bosch X, Guilabert A, Miquel R, Campo E. Enigmatic KikuchiFujimoto disease: a comprehensive review. Am J clin Pathol 2004;122:141-52. 
8. Adhikari RC, Sayami G, Lee MC, Basnet RB, Shrestha PK, Shrestha HG. Kikuchi-Fujimoto disease in Nepal: a study of 6 cases. Arch Pathol Lab Med 2003;127:1345-8.

9. Lin HC, Su CY, Huang CC, Hwang CF, Chien CY. Kikuchi's disease: a review and analysis of 61 cases. Otolaryngol Head Neck Surg 2003;128:650-3.

10. Jun-Fen F, Chun-Lin W, Li L, Dayan C, Guan-Ping D, Fung H. Kikuchi-Fujimoto disease manifesting as recurrent thrombocytopenia and Mobitz type II atrioventricular block in a 7- year-old girl: a case report and analysis of 138 Chinese childhood Kikuchi-Fujimoto cases with 10 years follow up in 97 patients. Acta Paediar 2007;96:1844-7.

11. Kikuchi M, Yoshizumi T, Nakamura H. Necrotizing lymphadenitis: possible acute toxoplasmic infection. Virchows Arch A Pathol Anat Histopathol 1977;376:247-53.

12. Feller AC, Lennert K, Stein H, Bruhn HD, Wuthe HH. Immunohistology and aetiology of histiocytic necrotizing lymphadenitis: report of three cases. Histopathology 1983;7:825-39.

13. Charalabopoulos $\mathrm{K}$, Charalabopoulos A, Papadopoulou $\mathrm{CH}$, Papalimneou V. Giardia lamblia intestinalis: a new pathogen with possible link to Kikuchi-Fujimoto disease. An additional element in the disease jigsaw. Int J clin Pract 2004;58:1180-3.

14. Unger PD, Rappaport KM, Strauchen JA. Necrotizing lymphadenitis (Kikuchi's disease). Report of four cases of an unusual pseudolymphomatous lesion and immunologic marker studies. Arch Pathol Lab Med 1987;111:1031-4.

15. Dorfman RF. Histiocytic necrotizing lymphadenitis of Kikuchi and Fujimoto [editorial]. Arch Pathol Lab Med 1987;111:1026-9.

16. Imamura $\mathrm{M}$, Ueno $\mathrm{H}$, Matsuura $\mathrm{A}$ et al. An ultrastructural study of subacute necrotizing lymphadenitis. Am J Pathol 1982;107:292-9.

17. Ohshima K, Shimazaki K, Kume T, Suzumiya J, Kanda M, Kikuchi M. Perforin and Fas pathways of cytotoxic T-cells in histiocytic necrotizing lymphadenitis. Histopathology 1998;33:471-8.

18. Yabe H, Sinzato I, Hashimoto K. Necrotizing lymphadenitis presenting as mesenteric lymphadenopathy. Rinsho Ketsueki 1999;40:658-62.

19. Yasukawa K, Matsumura T, Sato-Matsumura KC et al. Kikuchi's disease and the skin: case report and review of the literature. Br J Dermatol 2001;144:885-9.

20. Kapadia Y, Robinson BA, Angus HB. Kikuchi's disease presenting as fever of unknown origin. Lancet 1989;2:1519-20.

21. Yen A, Fearneyhough P, Raimer SS, Hudnall SD. EBV-associated Kikuchi's histiocytic necrotizing lymphadenitis with cutaneous manifestations. J Am Acad Dermatol 1997;36:342-6.

22. Kim SH, Kim SJ, Chung H, Lee HS, Kim HB, Park KH. Bilateral anterior uveitis as an unusual manifestation of Kikuchi-Fujimoto disease. Rheumatology 2004;43:1056-7.

23. Garcia-Zamalloa A, Taboada-Gomez J, Bernardo-Galan P, Magdalena FM, Zaldumbide- Duenas L, Ugarte-Maiztegui M. Bilateral pleural effusion and interstitial lung disease as unusual manifestations of Kikuchi-Fujimoto disease: case report and literature review. BMC Pulm Med 2010;10:54.
24. Douglas M, Bradbury R, Kannangara S, Mitchell D. Arthritis as an unusual manifestation of Kikuchi-Fujimoto disease. Rheumatology $2003 ; 42: 1010-2$.

25. Sumiyoshi Y, Kikuchi M, Ohshima K, Masuda Y, Takeshita M, Okamura T. A case of histiocytic necrotizing lymphadenitis with bone marrow and skin involvement. Virchows Arch A Pathol Anat Histopathol 1992;420:275-9.

26. Vasallo J, Coehlo Filho JC, Amaral VG. Histiocytic necrotizing lymphadenitis (Kikuchi lymphadenitis) in an HIV positive patient. Rev Inst Med Trop Sao Paulo 2002;44:265-8.

27. Charalabopoulos K, Papalimneou V, Charalabopoulos A, Bai M, Agnantis N. Brucella melitensis infection stimulates an immune response leading to Kikuchi-Fujimoto disease. In Vivo 2003;17:51-3.

28. Ramanan AV, Wynn RF, Kelsey A, Baildam EM. Systemic juvenile idiopathic arthritis, Kikuchi's disease and haemophagocytic lymphohistiocytosis - is there a link? Case report and literature review. Rheumatology 2003;42:596-8.

29. Belagavi CV, Kubeyinje EP. Recurrent cutaneous necrotizing vasculitis in a patient with Kikuchi's disease: case report. East Afr Med J 1997;74:747-8.

30. Wong CY, Law GT, Shum TT, Wong KY, Li YK. Pulmonary hemorrhage in a patient with Kikuchi disease. Monaldi Arch Chest Dis 2001;56:118-20.

31. Martinez-Vazquez C, Huqhes G, Bordon J et al. Histiocytic necrotizing lymphadenitis, Kikuchi-Fujimoto's disease associated with systemic lupus erythematosus. QJM 1996;90:531-3.

32. Keogh MA, Williamson RM, Denaro CP. Kikuchi's disease associated with parotidomegaly, thyroiditis and a rash in a young man. Aust $\mathrm{N}$ Z J Med 2000;30;633-4.

33. Radhi JM, Skinnider L, McFadden A. Kikuchi's lymphadenitis and carcinoma of the stomach. J Clin Pathol 1997;50:530-1.

34. Yoshino T, Mannami T, Ichimura $\mathrm{K}$ et al. Two cases of histiocytic necrotizing lymphadenitis (Kikuchi-Fujimoto disease) following diffuse large B-cell lymphoma. Hum Pathol 2000;31:1328-31.

35. Dominguez DC, Torres ML, Antony S. Is human herpesvirus 6 linked to Kikuchi-Fujimoto disease? The importance of consistent molecular and serologic analysis. South Med J 2003;96:226-33.

36. Osborn M, Aqel N, Levine TS. The fine needle aspiration appearances of Kikuchi's lymphadenitis. Cytopathology 2009;20:36-43.

37. Kucukardali Y, Solmazgul E, Kunter E, Oncul O, Yildirim S, Kaplan M. Kikuchi- Fujimoto disease: analysis of 244 cases. Clin Rheumatol 2007;26:50-4.

38. Hutchinson CB, Wang E. Kikuchi-Fujimoto disease. Arch Pathol Lab Med 2010;134:289-93.

39. O'Nell D, O' Grady J, Variend S. Child fatality associated with pathological features of histiocytic necrotizing lymphadenitis (Kikuchi-Fujimoto disease). Pediatr Pathol Lab Med 1998;18:79-88.

40. Takada K, Suzuki K, Hidaka T et al. Immediate remission obtained by minocycline in a patient with histiocytic necrotizing lymphadenitis. Intern Med 2001;40:1055-8. 